\title{
Nesobasis rito sp. nov. (Zygoptera: Coenagrionidae), a new species of forest damselfly from Vanua Levu, Fiji
}

\author{
ANAIS RIVAS-TORRES ${ }^{1,6}$, BINDIYA RASHNI ${ }^{2,7}$, HILDA WAQA-SAKITI I, , MARIKA TUIWAWA ${ }^{4}$, \\ MARÍA OLALLA LORENZO-CARBALLA ${ }^{1,9}$, CHRISTOPHER D. BEATTY $^{5,10}$ \\ \& ADOLFO CORDERO-RIVERA ${ }^{1, *}$ \\ ${ }^{1}$ Universidade de Vigo, ECOEVO Lab., Escola de Enxeñaría Forestal, Campus Universitario, 36005 Pontevedra, Spain \\ ${ }^{2}$ NatureFiji-MareqetiViti, P. O. Box 2041, Government Buildings, Suva, Fiji \\ ${ }^{3}$ Pacific Center for the Environment \& Sustainable Development, The University of the South Pacific, Laucala Campus, Suva, Fiji \\ ${ }^{4}$ South Pacific Regional Herbarium, Biodiversity Centre, Institute of Applied Science, The University of the South Pacific, Laucala \\ Campus, Suva, Fiji \\ ${ }^{5}$ Center for Comparative and Population Genomics, Department of Ecology \& Evolutionary Biology, Cornell University, Ithaca, New \\ York 14853, USA \\ ${ }^{6} \odot$ https://orcid.org/0000-0002-5527-0564 \\ 7 ○ttps://orcid.org/0000-0002-7699-9549 \\ 8 (ㄴ https://orcid.org/0000-0002-3781-8928 \\ 9 ๑ https://orcid.org/0000-0003-4570-2594 \\ ${ }^{10} \odot$ https://orcid.org/0000-0003-2641-0000 \\ ${ }^{*}$ Corresponding author. (1) adolfo.cordero@uvigo.gal; @ ittps://orcid.org/0000-0002-5087-3550
}

\begin{abstract}
Nesobasis rito sp. nov. (Holotype $\widehat{\jmath}$, Fiji, Vanua Levu, Drawa, 31 v 2018, A. Rivas-Torres leg.) from the comosa group is here described, illustrated, diagnosed, and compared with morphologically close species of the genus. Nesobasis rito can be distinguished from its related congeners by the shape of the caudal appendages and the ligula. The most similar species are $N$. comosa and $N$. heteroneura, which, like $N$. rito, have the caudal appendages covered by dense setae (especially the first species), but the shape differs clearly in lateral view, with $N$. rito having longer and more slender appendages, and a basal tooth clearly seen in dorsal view, absent in other members of the comosa group. The specific status of the collected specimens is also supported by the results of genetic analyses, where $N$. rito appears as a well-supported monophyletic clade. Nesobasis rito also has a distinct distribution from its most similar congeners: it is found on Vanua Levu, while $N$. comosa is found on Viti Levu and the closely related $N$. heteroneura is found on Viti Levu and Ovalau. All species of this group are found in streams with native forest riparian vegetation on their respective islands.
\end{abstract}

Key words: Odonata, Zygoptera, Nesobasis, taxonomy, Vanua Levu, Fiji

\section{Introduction}

The genus Nesobasis was erected by Selys (1891) to include specimens collected at the islands "Viti", with a general appearance similar to the genus Pseudagrion Selys, 1876, but lacking postocular spots, having longer setae in the legs, the lower tooth of the claws almost as long as the upper, the upper anal appendages of males not forked or notched at the end, and a simple prothorax. Later, Tillyard (1924) compiled the knowledge of the odonates of Fiji, describing 13 species, ten in the genus Nesobasis, one of which was placed in Melanesobasis by Donnelly (1984). Kimmins (1943) described N. leveri from a single male collected on Viti Levu. Marinov (2021) introduced N. martina and N. monika found on Viti Levu and Taveuni, respectively.

Donnelly (1990) reviewed in detail the fauna of Viti Levu, Ovalau and Kadavu, which he divided into three groups, while also describing ten additional species, and indicating that, taking into account the undescribed species from other Fijian islands, this genus is the most speciose example of an odonate island radiation. The results of an extensive study corroborated this fact, with the finding of at least 24 species, including two additional undescribed 
species (Beatty et al. 2007). Currently 23 species are described, but 13 more are being prepared for description (Beatty et al. 2017; T. Donnelly and M. Marinov, pers. comm.).

Several biological attributes make Nesobasis a very unusual genus compared to other coenagrionids. Donnelly (1990) was the first to highlight that some species of Nesobasis have a female-biased sex-ratio and he attributed this fact to a sex-role reversal, with territorial females on the streams and males, which are rarely encountered, remaining away from the water. However, sex-role reversal was not found when the behaviour of these species was studied in detail, although for three species male rarity is extreme, and in at least two species males are still unknown (Van Gossum et al. 2007). Parthenogenesis has been suggested as the reproductive mode for these female-biased species of Nesobasis, but until now the only confirmed case of parthenogenesis in the Odonata remains the Azorean populations of another coenagrionid, Ischnura hastata (Say, 1840) (Lorenzo-Carballa \& Cordero-Rivera 2009).

Nesobasis is also remarkable because the two main islands, Viti Levu and Vanua Levu, have similarly rich fauna, but do not share any species, whereas smaller islands around them have a subset of the species from the largest islands, making the biogeography of Nesobasis a fascinating example of island radiation (Beatty et al. 2017; Van Gossum et al. 2008). Only one island has species from both assemblages: the island of Koro, located between Viti Levu and Vanua Levu, has a single species from the former island (N. rufostigma Donnelly, 1990) along with 3 species from the latter ( $N$. brachycerca Tillyard, 1924 and two undescribed species, Van Gossum et al. 2008).

Nesobasis is the dominant genus of damselflies found in Fiji, showing wide variation in size, coloration, and morphology (Donnelly 1990), but several species are commonly found in the same stream (as many as 8-12), with apparently little ecological divergence (Beatty et al. 2017; Van Gossum et al. 2007). Many species of Nesobasis have been found to be infected with a variety of strains of Wolbachia (Lorenzo-Carballa et al. 2019), a bacterium known to influence sex ratios and mate compatibility in several arthropod hosts (Bruzzese et al. 2021; Werren et al. 2008). While evidence for parthenogenesis in Nesobasis is lacking, there is a possible influence of Wolbachia infection on the speciation rate of these damselflies (Lorenzo-Carballa et al. 2019). Comparative phylogenetic studies and further analyses of the Wolbachia infections are needed to understand whether and how these endosymbionts, paired with geographical isolation, have contributed to the high diversity in this genus (Beatty et al. 2017; LorenzoCarballa et al. 2019). However, such a task cannot be completed until all the species within the genus are described. To contribute to this goal, we here describe a new species of Nesobasis from Vanua Levu.

\section{Material and methods}

Specimen collection and morphological analyses. Specimens were collected during surveys of odonates in different localities of the island of Vanua Levu, in September 2005, August 2009, September 2011 and May-June 2018.

Given the large number of Nesobasis species currently awaiting description, we consulted an unpublished key from M. Marinov and notes from T.R. Donnelly, to determine whether the collected specimens were already included in their on-going studies. These specimens were found to be novel. To document the new species, field photographs were taken with a Canon EOS 5D Mark II and 7D Mark II with a Canon $100 \mathrm{~mm}$ macro lens. Laboratory photographs were taken with a Leica Flexacam C1 camera attached to an Olympus SZ60 stereomicroscope, and stacked with Adobe Photoshop CS6 to increase depth of field. Specimens were immersed in ethylene glycol to diminish bright areas in the pictures, allowing a sharper view of structures and increasing the contrast between pale and dark areas.

We follow Garrison et al. (2010) for body morphology nomenclature. Total body length includes cerci; all measurements are given in $\mathrm{mm}$. Abbreviations for structures used throughout the text are as follows: S1-10: abdominal segments 1 to 10, Fw: forewing, Hw: hindwing.

Specimens are preserved at:

ECOEVO Laboratory of Evolutionary and Conservation Ecology, Escola de Enxeñaría Forestal, University of Vigo, Pontevedra, Spain.

NZAC New Zealand Arthropod Collections, Auckland, New Zealand

USP The University of the South Pacific, Suva, Fiji. 


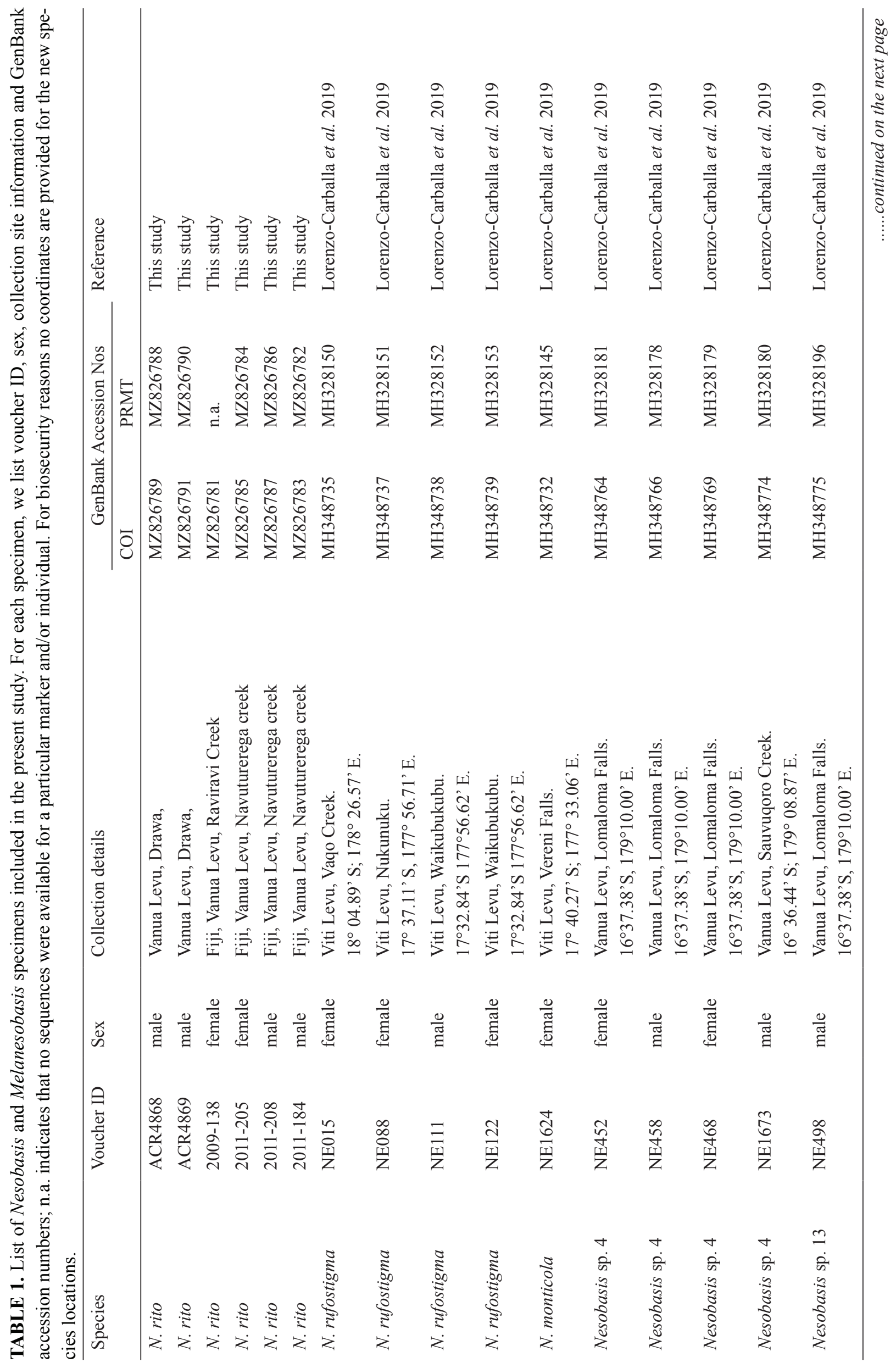




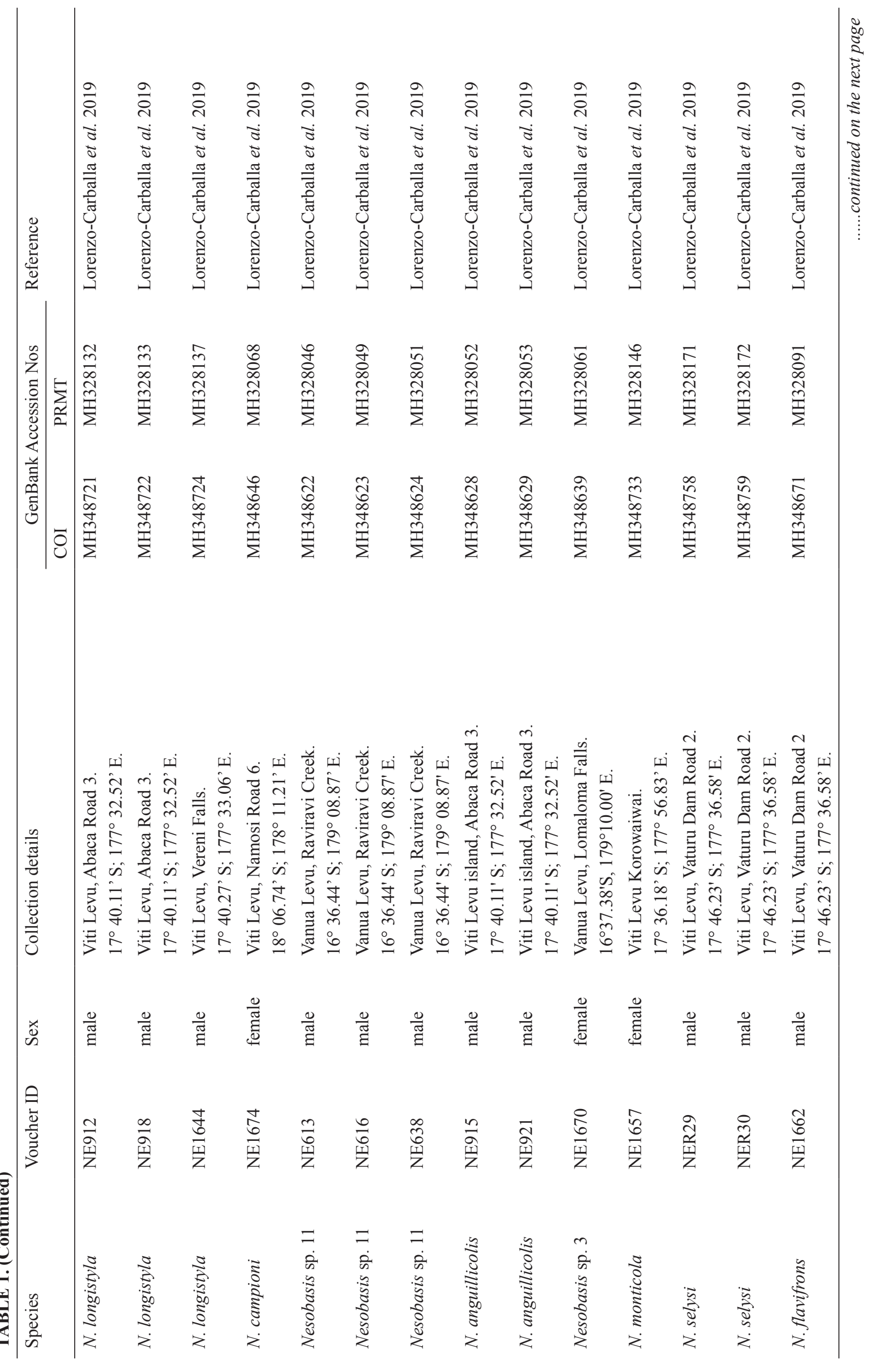




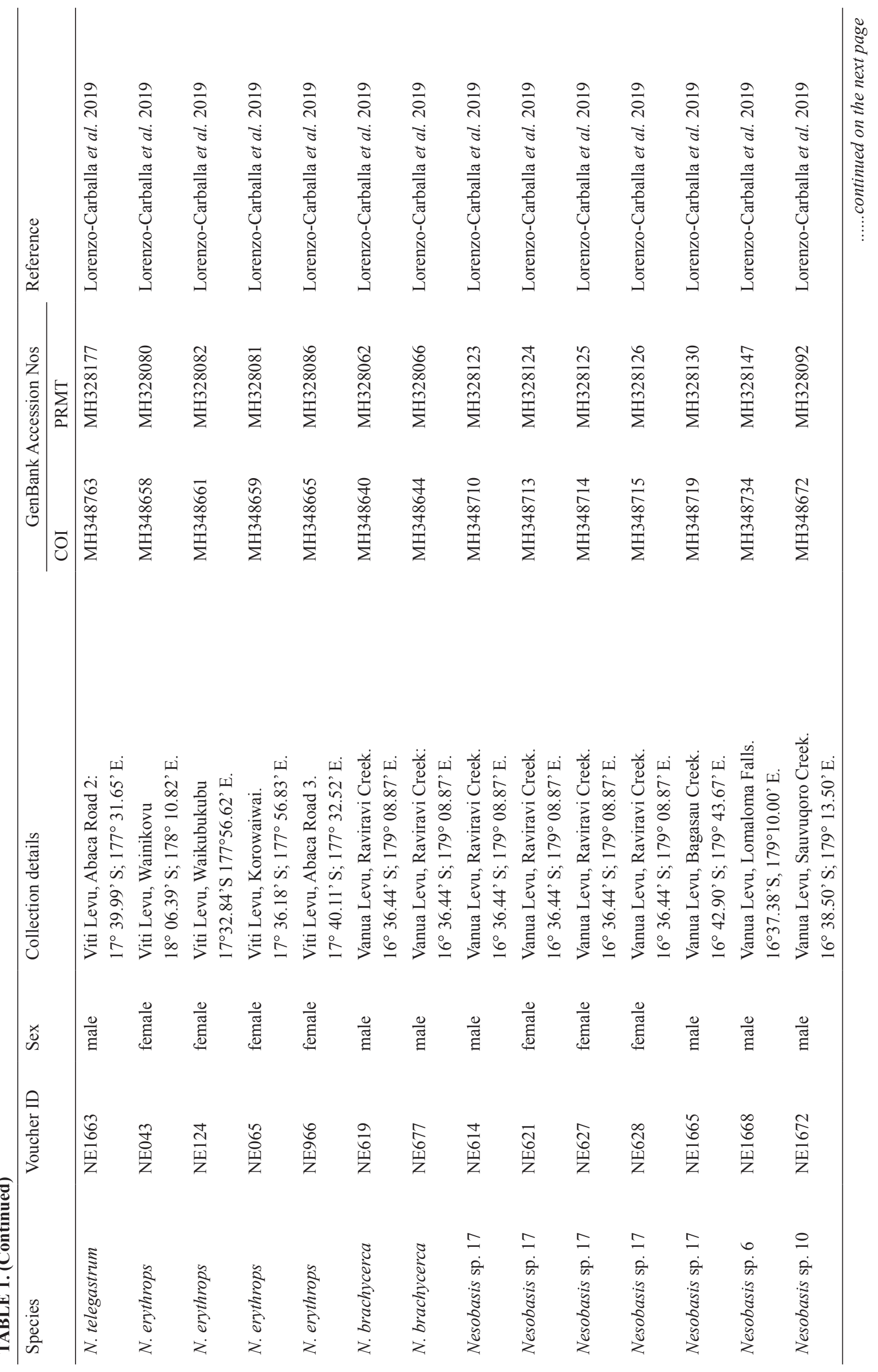




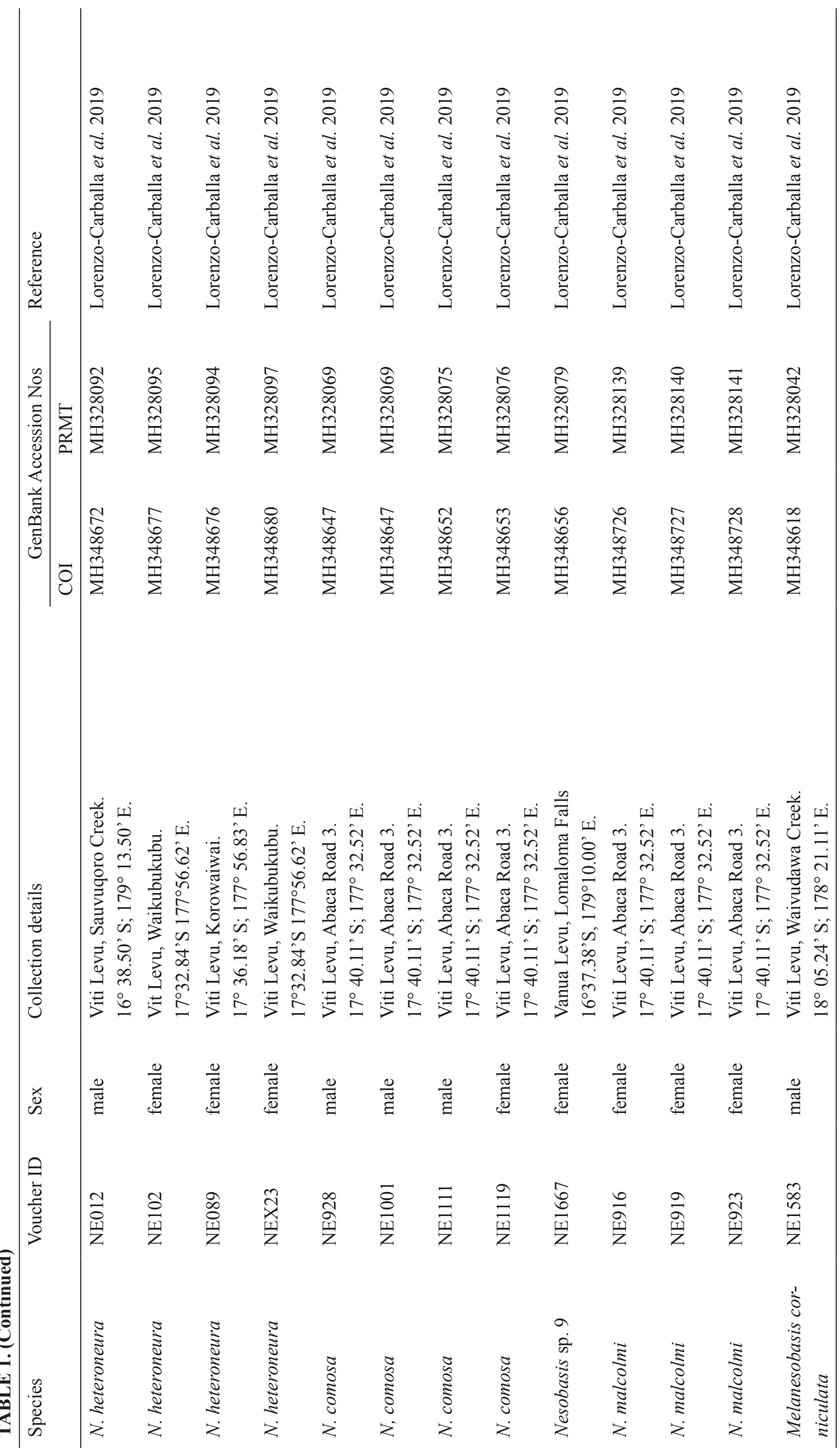


DNA extraction and sequencing. Among the adults captured, six specimens of the putative new Nesobasis species ( $4 \hat{\delta} \widehat{\delta}$ and 2 우), were selected for genetic analyses (see Table 1). Adult individuals were captured using a hand net, and preserved in $80 \%$ ethanol prior to DNA extraction. Total genomic DNA was extracted from a single leg of each specimen using the GeneJet DNA extraction kit (ThermoFisher Scientific, Waltham, MA, USA), following the manufacturer's protocol. We amplified a fragment of the mitochondrial Cytochrome Oxidase I (COI) gene, and the nuclear Arginine Methyltransferase (PRMT) gene, using previously published primers (Ferreira et al. 2014; Futahashi 2011) (Table 2). PCR reactions were carried out at specific annealing temperatures (Table 2) using the DreamTaq Green PCR Master Mix (ThermoFisher Scientific). Prior to sequencing, unincorporated primers and dNTPs were removed using Shrimp Alkaline Phosphatase and Exonuclease I (New England Biolabs, Ipswich, MA, USA). Cleaned PCR products were sequenced in both directions using BigDye v.3.1 chemistry (Applied Biosystems, Foster City, CA, USA) in an ABI 3730xl DNA Analyzer (Applied Biosystems), at the Macrogen Spain facilities in Madrid.

TABLE 2. Primers used to amplify mitochondrial (COI) and nuclear (PRMT) DNA of the Nesobasis species included in this study. Ta indicates the PCR annealing temperature for each primer pair.

\begin{tabular}{lllll}
\hline Locus & Primer name & Primer sequence $\left(5^{\prime}-3{ }^{\prime}\right)$ & Ta $\left({ }^{\circ} \mathrm{C}\right)$ & Reference \\
\hline Cytochrome oxidase I & COI-S0 & TACCAATTATAATTGGAGGATTYGG & 48 & Futahashi 2011 \\
(COI) & COI-AS0 & CTTCTGGATGTCCAAARAATCA & 48 & Futahashi 2011 \\
\hline $\begin{array}{l}\text { Arginine methyltrans- } \\
\text { ferase (PRMT) }\end{array}$ & ARG-F2 & TGCCGCCAAGGCTGGAGCATC & 48 & Ferreira et al 2014 \\
\hline
\end{tabular}

Genetic analyses. After sequencing, chromatograms were visually inspected, trimmed and assembled using Geneious v. 9.1.7 (https://www.geneious.com). BLAST searches were run through Geneious v. 9.1.7 for all DNA sequences, to ensure that they were not derived from contamination. Previously published sequences from several Nesobasis species were downloaded from GenBank (https:/www.ncbi.nlm.nih.gov/genbank/) and added to our datasets. Sequences of Melanesobasis corniculata (Tillyard, 1924) were selected as outgroups for the phylogenetic analyses. Sequences of the species described in the present study have been deposited in GenBank with accession numbers indicated in Table 1.

Sequences were aligned using MAFFT (Katoh et al. 2002) as implemented in Geneious v. 9.1 .7 (https://www. geneious.com). Alignments were exported into MEGA X (Kumar et al. 2018) for estimation of genetic differentiation between the Nesobasis species. Genetic p-distances were estimated with the pairwise deletion option, which removes all ambiguous positions for each sequence pair. Phylogenetic relationships among Nesobasis were reconstructed using maximum likelihood (ML) and Bayesian inference (BI) approaches. ML analyses were carried out using RAxML 7.2.8. (Stamatakis 2006) as implemented in Geneious v. 9.1.7, using the rapid bootstrapping and search for best scoring ML tree option, under the GTR $+\mathrm{G}+\mathrm{I}$ model. Support for the nodes in the resulting tree was estimated by running 1,000 bootstrap replicates. BI analyses were conducted using MrBayes 3.2.6 (Huelsenbeck \& Ronquist 2001; Ronquist \& Huelsenbeck 2003) also implemented in Geneious v. 9.1.7. Searches were run for 1.1 million generations, with default priors and the GTR+G+I substitution model.

\section{Results}

\section{Nesobasis rito sp. nov. Rivas-Torres \& Cordero-Rivera}

(Figs. 1-3)

Holotype. $\widehat{\delta}$ (currently in ECOEVO, to be deposited at NZAC, accession number NZAC04231068; specimen code ACR-04869), Fiji, Vanua Levu, Drawa, Drawa River, altitude 52 m, collected on 31.v.2018, A. Rivas-Torres leg. Paratypes. $\delta$ (currently in ECOEVO, to be deposited at USP, specimen code ACR-04868), Fiji, Vanua Levu, Drawa, Drawa River (same data as holotype), collected on 31.v.2018. A. Rivas-Torres leg.; $\widehat{\partial}$ (ECOEVO, specimen code 2011-184, Fiji, Vanua Levu, Navuturerega creek, C. Beatty leg.), collected on 16.ix.2011; $\partial^{\Uparrow}$ (ECOEVO, specimen code 2011-208, Fiji, Vanua Levu, Navuturerega creek, C. Beatty leg.), collected on 16.ix.2011.

Paratypes. $\odot$ (currently in ECOEVO, to be deposited at NZAC, accession number NZAC04231069; specimen 
code 2009-138), Fiji, Vanua Levu, RaviRavi, C. Beatty leg., collected on 7.viii.2009; + (ECOEVO, specimen code 2011-205, Fiji, Vanua Levu, Navuturerega creek, C. Beatty leg.), collected on 16.ix.2011.

Etymology. Named rito (noun in apposition) which in Spanish means ritual, to honour the local community rituals of Drawa village, which we enjoyed before finding the specimens, but also as an acronym of Rivas-Torres, as an appreciation of the first author to her family's continuous support.

Description of holotype. Figure 1 shows the habitus of the dried specimen, and the dorsal view of the last abdominal segments. Figure 5 illustrates several live individuals in the field.

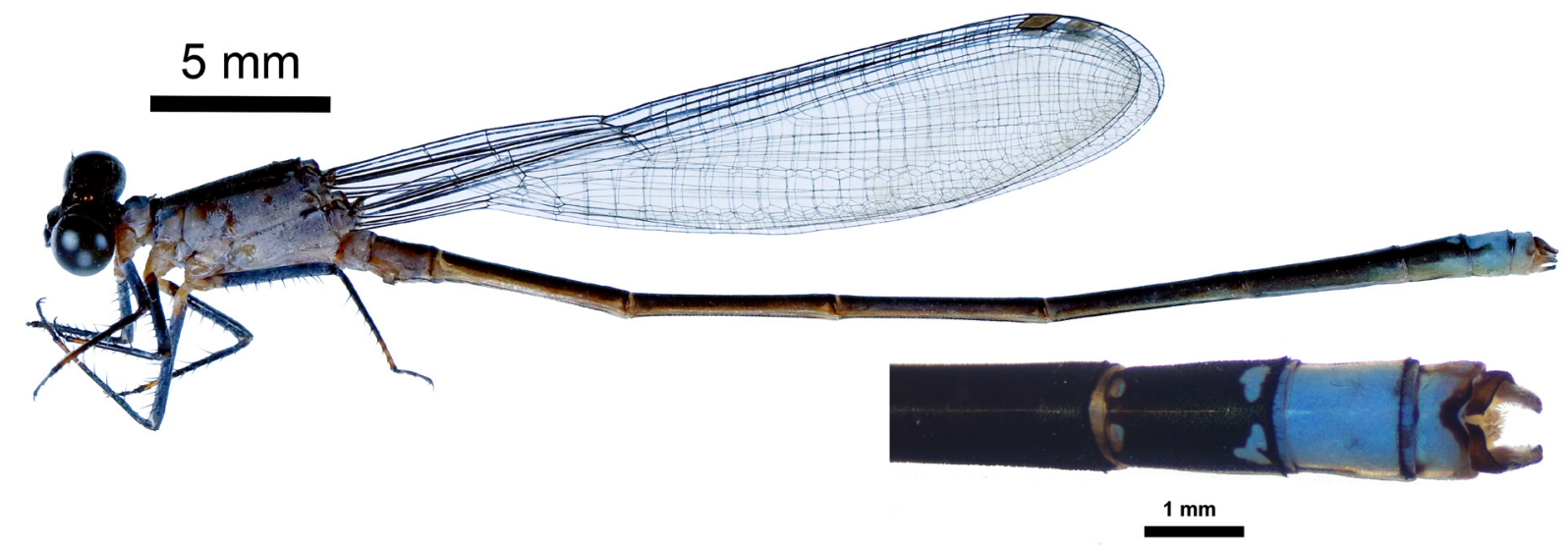

FIGURE 1. Nesobasis rito sp. nov., holotype male; habitus and dorsal view of S7-10, showing the blue spots and the caudal appendages.

Head. (Fig. 2a, c). Epicranium totally black; labrum pale blue, anteclypeus dark brown and postclypeus black; antefrons pale brown, and postfrons black; without postocular spots; antennae, occipital bar and border of postocular lobes black. Eyes pale green in life, dark brown in dried specimens but pale grey when immersed in ethylene glycol (Fig. 2b). Mandibles pale brown (Fig. 2c).

Thorax. (Fig 2b). Prothorax largely black, except anterior and posterior lobes, which have a pale brown bar, the same colour as the propleuron. In dorsal view, the posterior lobe appears slightly bilobed (Fig. 2a). Mesothorax with mesepisternum completely black, mesepimeron pale brown with a black longitudinal stripe occupying about half of its width, metepisternum pale brown with a small dark streak on the posterior end, and a small line at the posterior ventral border; metepimeron pale brown. The pale areas of the thorax are blue in life. In the dried specimen there is a bluish pruinescence covering the sides of the thorax (Fig. 1). Legs mainly pale black, with the coxae and trochanters pale brown, and the last third of tibiae reddish (Fig. 1).

Wings. (Fig. 2f). Hyaline, venation dark, 14 antenodals, pterostigma brown, about $0.8 \mathrm{~mm}$ width. A single line of cells distal of pterostigma.

Abdomen. (Fig. 1). Mostly black, S1 and S2 black dorsally, pale laterally (bluish alive, brown post-mortem); S3-6 black dorsally and pale ventrally; S7 black and pale ventrally, but with a blue ventral part, covering the last quarter; S8 black dorsally with two blue irregular spots posteriorly and two blue dots in the anterior part, blue laterally; S9 and S10 almost blue, with a dark area in the distal part of S10 dorsally.

Caudal appendages. (Fig. 2e, g). In dorsal view, only the cerci are clearly visible, convergent, with a terminal tooth and a small basal tooth (arrow in Fig. 2e) and covered with a dense pale pilosity. In lateral view, cerci and paraprocts slightly longer than S10. Cerci dark brown, oriented downwards and with rounded end. Paraprocts light brown basally and dark brown distally, almost horizontal, with rounded tip.

Genital ligula. (Fig. 3). Distal segment simple, similar to a spoon, without flagella, but with an indentation that creates a rounded lateral lobe in each side.

Measurements. Body length: 41.2, Hw: 23.8.

Male paratypes. The paratypes are very similar to the holotype. Specimen ACR-04868 has the general appearance of a younger specimen. The pale areas of thorax are bluish in life (as shown for other specimens in Fig. 5a-c) and the black stripe of mesepimeron broader, fused with the black mesepisternum (Fig. 2d). The distal blue spots in S8 are larger than in the holotype and drop-shaped (Fig. 2i). Caudal appendages very similar to holotype (Fig. 2h). Blue spots on S8 are reduced in specimen 2011-184. Specimen 2011-208 shows some pruinescence on the last abdominal segments, indicating it is an older individual. 


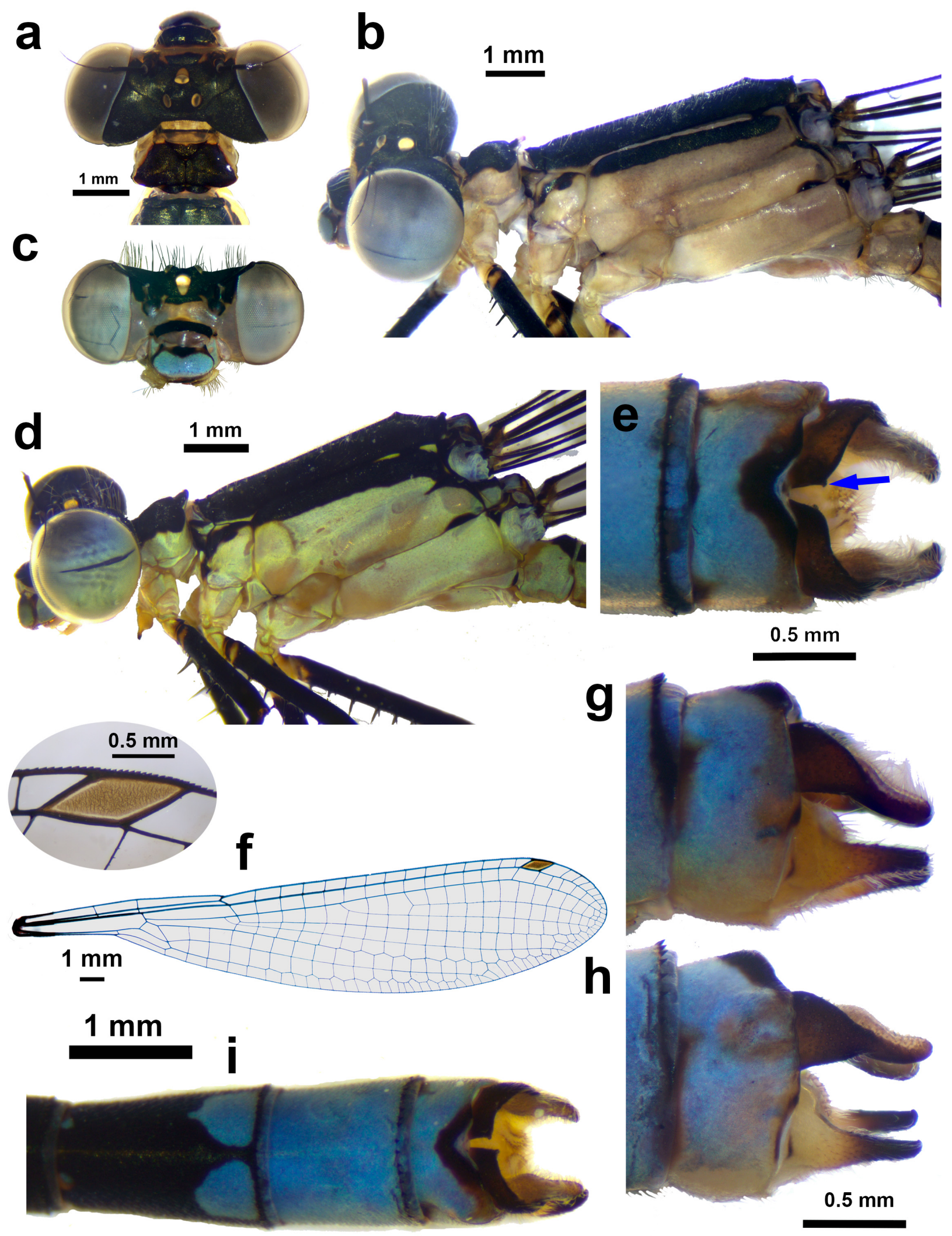

FIGURE 2. Nesobasis rito sp. nov., holotype male from Vanua Levu, Fiji: (2a) head in dorsal view. Note the notch at posterior lobe of prothorax; (2b) lateral view of the thorax; (2c) head in frontal view; (2e) caudal appendages in dorsal view, with the basal tooth of the cerci indicated by the arrow; (2g) caudal appendages in lateral view; (2f) hw and a detail of the pterostigma; paratype male: (2d) lateral view of head and thorax, showing extended black pattern on the mesepimeron compared to the holotype; (2h) lateral view of the caudal appendages; (2i) dorsal view of S8-10, showing large blue spots in S8. 


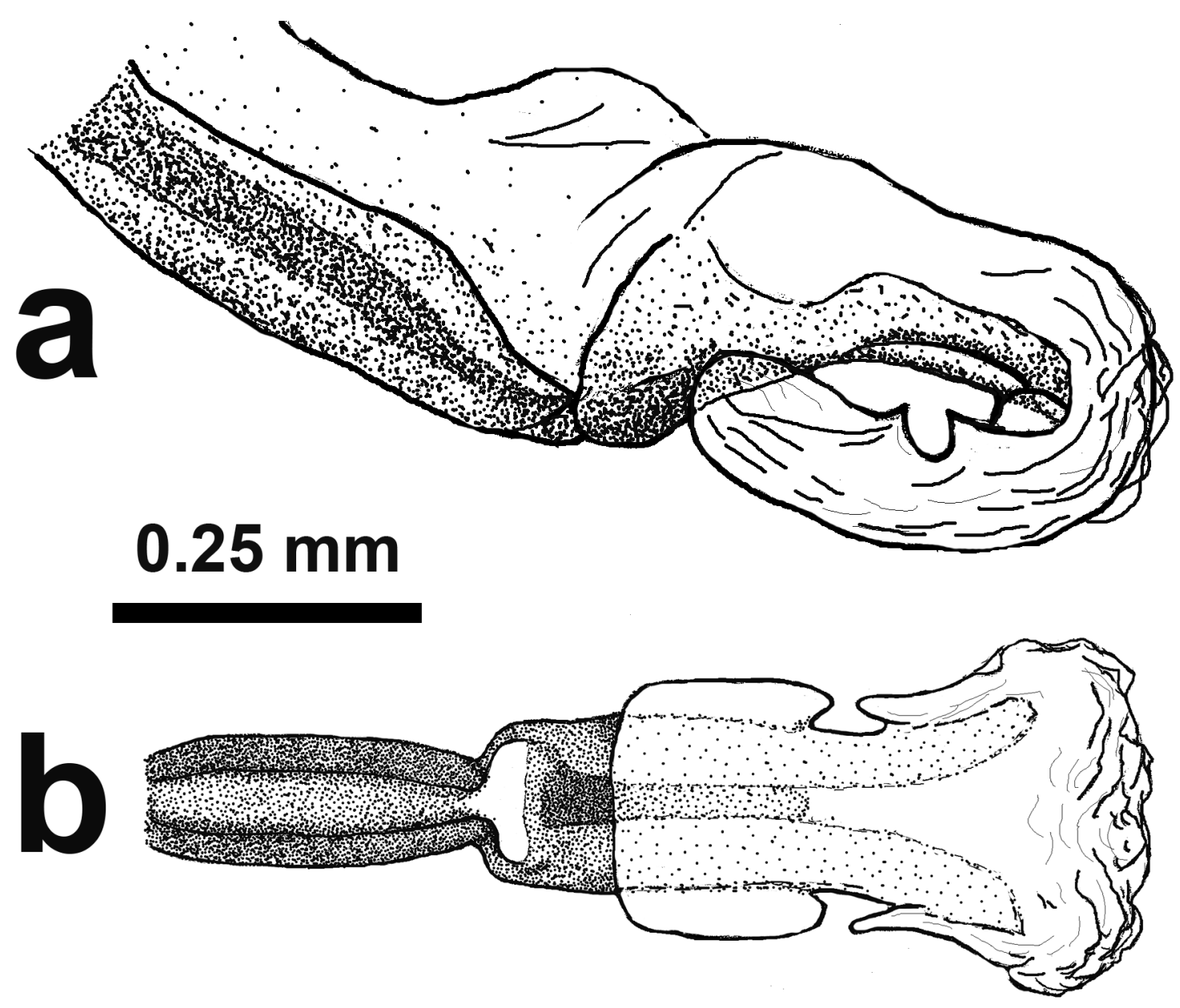

FIGURE 3. Nesobasis rito sp. nov., holotype male from Vanua Levu, Fiji; (3a) lateral view of the genital ligula; (3b) ectal view.

Measurements. Specimen ACR-04868: Body length: 40.8, Hw: 23.8; specimen 2011-184: Body length: 40.2, Hw: 21.4; specimen 2011-208: Body length: 40.8, Hw: 22.0.

Female paratypes: Very similar to the male in general appearance (Fig. 4). The pale areas of the body were reddish in life (Fig. 5d-e) but pinkish in the preserved specimens (Fig. 4a, f). The pronotum is trapezoidal in dorsal view, with two small triangular expansions on the frontal border. The posterior lobe with a narrow elevated tubercle in lateral view (Fig. 4f). The valves of the ovipositor reach almost to the tip of the cerci (Fig. 4c, d). The pterostigma is uniformly light brown (Fig. 4e).

Measurements. Specimen 2009-138: Body length: 36.2; specimen 2011-205: Hw: 22.6; body length: 37.5, Hw: 22.8 .

\section{Discussion}

Differential diagnosis. According to its morphology, $N$. rito belongs to the comosa group as defined by Donnelly (1990), which includes N. comosa, N. heteroneura, N. aurantiaca, N. malcolmi and N. martina. Based on the phylogeny of Beatty et al. (2017) this is a highly supported clade. One unique feature of $N$. rito is a notch at around the middle from the posterior lobe of the prothorax that makes it somewhat bilobed (Fig. 2a). Nesobasis rito differs markedly in its appendages from $N$. malcolmi and $N$. aurantiaca, two species that have very short caudal appendages. Nesobasis rito resembles $N$. comosa because the caudal appendages are covered by dense setae, although their shape differs clearly in lateral view: $N$. rito has longer and more slender appendages, and a basal tooth that can be seen in dorsal view (Fig. 2e) which is absent in $N$. comosa. The general body colouration and the cerci resemble also those of $N$. heteroneura, although, in $N$. rito, the cerci are longer, with more pilosity and differ also by the pres- 
ence of the basal teeth. Furthermore, the blue spots of S8 are not mentioned in the description of $N$. heteroneura or $N$. comosa. The genital ligula in lateral view (Fig. 3a) is similar to that of $N$. comosa, but with the distal segment broader and the lateral lobes more pronounced (compared to Fig. 103, Donnelly, 1990). In ectal view (Fig. 3b) more elongated than the ligula of $N$. comosa and the lateral lobes clearly visible (compared to Fig. 104, Donnelly, 1990). Like $N$. heteroneura, the wings of $N$. rito have a single row of cells distal from the pterostigma but in $N$. comosa wings have mostly double rows of cells (M. Marinov, personal communication).
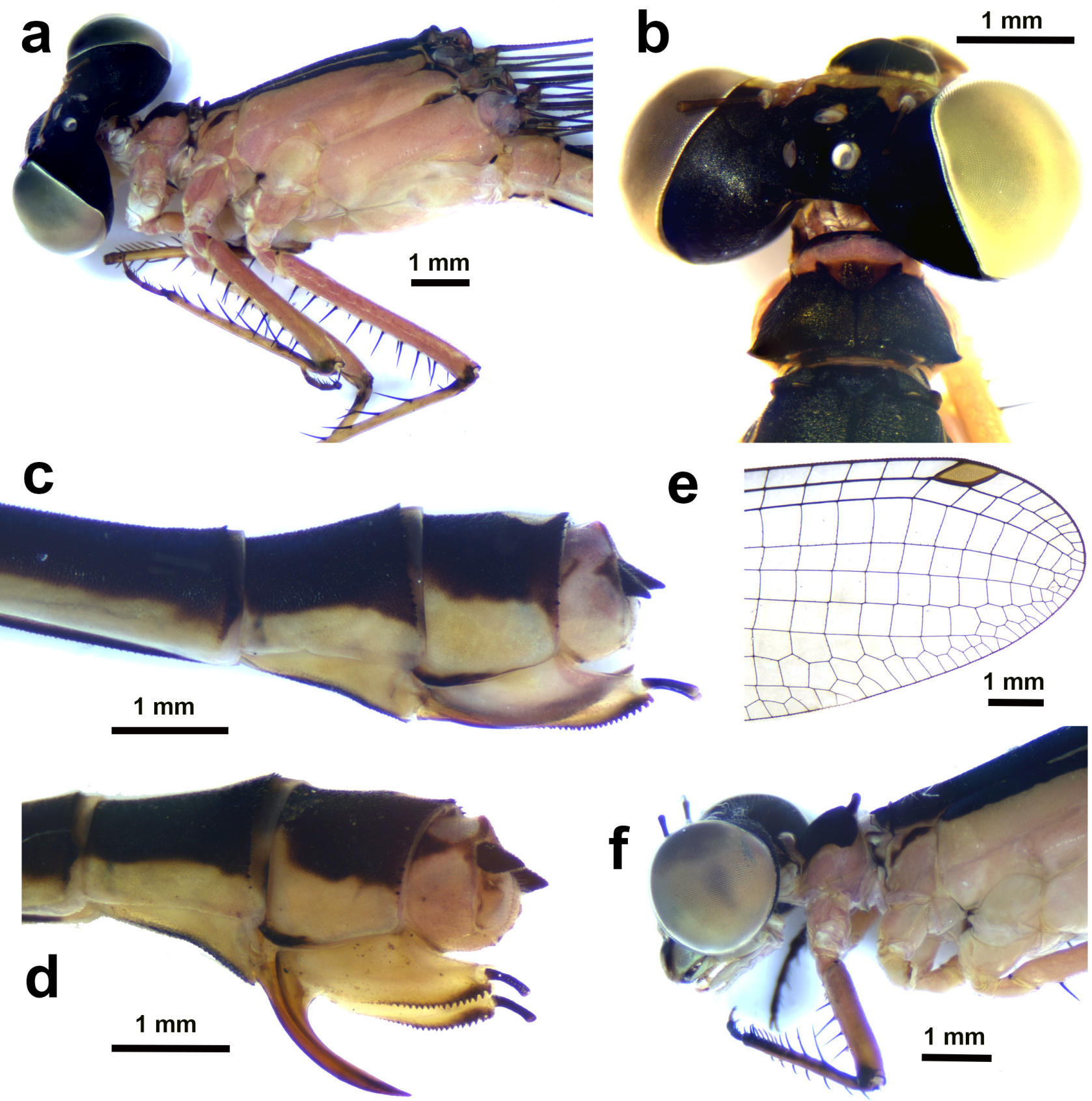

FIGURE 4. Nesobasis rito sp. nov., paratype females from Vanua Levu, Fiji; (4a) female 2009-138, lateral view of the head and thorax; (4b) dorsal view of the head and the pronotum; (4c) S7-10 in lateral view; (4e) detail of the left hindwing; (4d) S8-10 of female 2011-205, showing the stylus; (4f), lateral view of the head and prothorax of female 2011-205.

It is of note that specimens of $N$. comosa and $N$. heteroneura resemble one another; while generally $N$. comosa males have more dense setae on their caudal appendages (similar to $N$. rito) and are somewhat more robust, these traits can be variable, making it difficult to resolve $N$. comosa and $N$. heteroneura in some locations. In the phylogenies of Beatty et al. (2017), Lorenzo-Carballa et al. (2019) and in our genetic results here (Figs. 6 and 7, Table 3), a close relationship between these species is suggested. It is possible that $N$. comosa and $N$. heteroneura represent 
a species complex; it has been noted that specimens more closely resembling $N$. comosa are found at higher elevation localities, with more characteristic $N$. heteroneura individuals appearing in lower-elevation streams (CDB pers. obs.). Thus, these two species may represent two morphotype extremes along a habitat gradient, a hypothesis that needs further research.
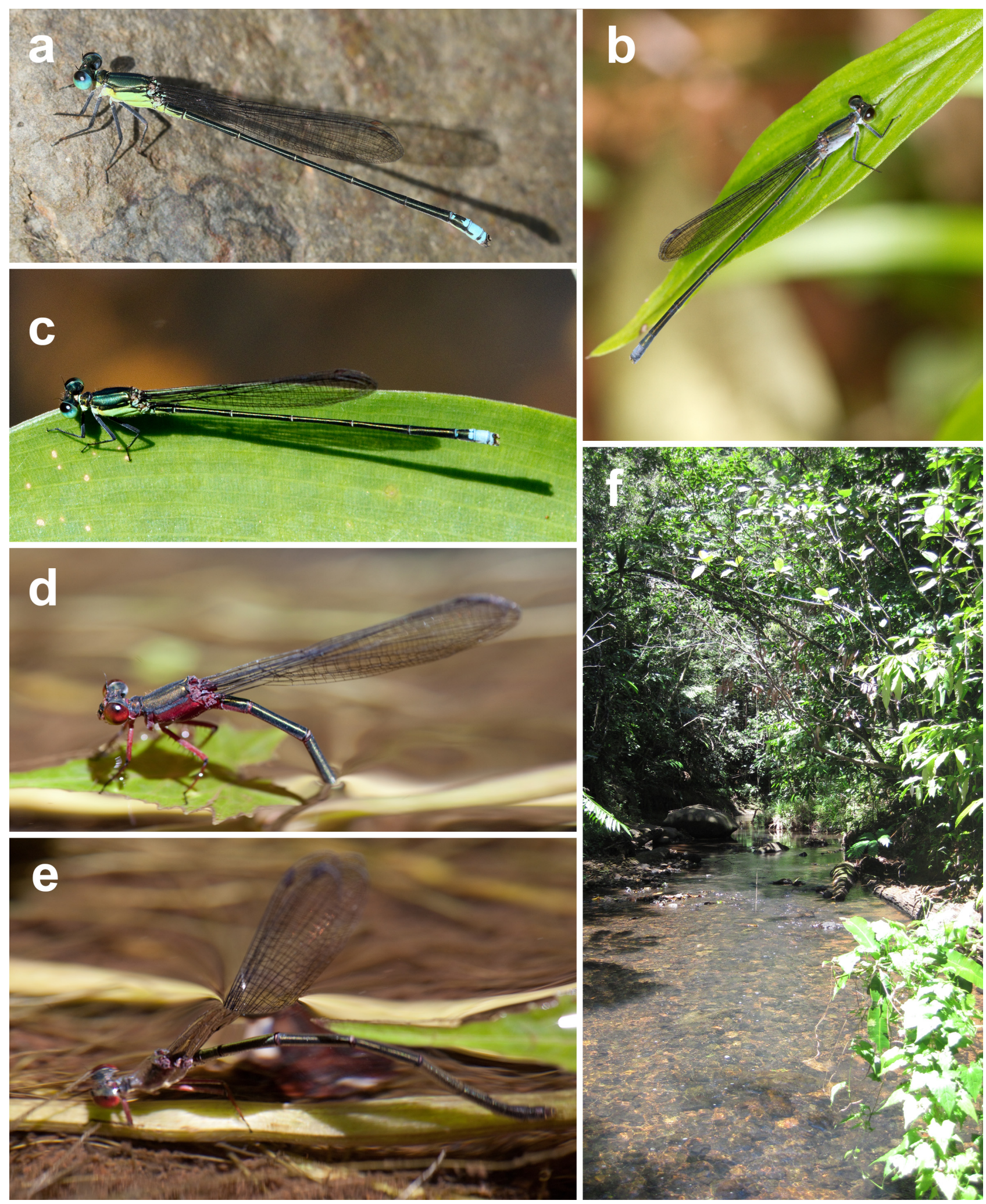

FIGURE 5. Live specimens of Nesobasis rito sp. nov. and habitat. (5a) A young male from the type locality, Drawa River; (5b) a pruinescent male and a mature male (5c) from Navuturerega creek; (5d-e) a female laying eggs in the Navuturerega creek, a section of which is shown in (5f). Pictures by AC-R. 
Habitat and ecology. The species was found in shallow but wide streams (about 4-5 m wetted channel width, Fig. 5f), commonly in shaded areas, with native riparian vegetation, but in the open as well. Males perched both in the vegetation (Fig. 5b, c) and on stones in the middle of the stream (Fig. 5a). Females lay eggs alone and may go underwater to oviposit (Fig. 5d, e).

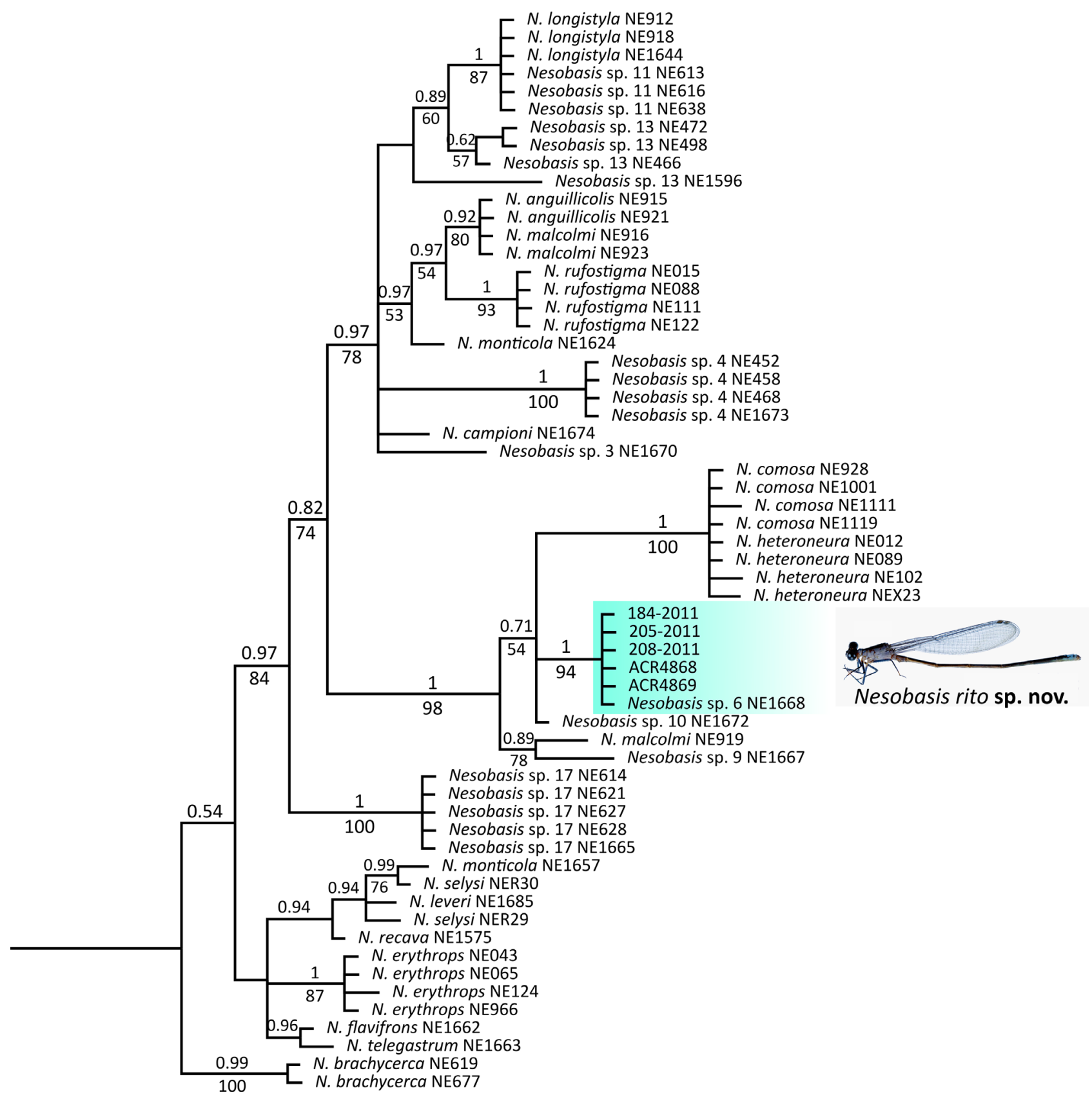

FIGURE 6. Phylogenetic tree representing the relationships among the Nesobasis species included in the present study for the PRMT nuclear marker. The tree illustrates the position of Nesobasis rito sp. nov. within the comosa group. Values above/below branches represent BI posterior probability and ML bootstrap support values, respectively. Only bootstrap support values $\geq 50 \%$ are shown. Melanesobasis corniculata was used as the outgroup in these analyses (see main text and Table 1 for details) but for the purposes of illustration, only the ingroup (i.e. Nesobasis) is shown here.

Genetic relationships with other Nesobasis species. The genetic analyses showed that, for the nuclear DNA marker, $N$. rito sp. nov. appears as a well-supported, monophyletic clade, within the comosa group (Figs. 6-7). For the mitochondrial DNA, some discordance with the nuclear DNA phylogeny is observed, regarding the placement of $N$. rito in relation to the clade that includes $N$. heteroneura and $N$. comosa (Fig. 7). These results are likely due to the widespread Wolbachia infections that are found within the species of Nesobasis (Lorenzo-Carballa et al. 2019). Yet, the fact that the specimens of $N$. rito appear in both cases as a well-supported, monophyletic clade, provides 
strong support for the distinctiveness of these specimens and corroborates the results of the morphological analyses. In agreement with the phylogenetic analyses, the genetic distances between $N$. rito and $N$. heteroneura and $N$. comosa are the lowest for both sequenced markers (Table 3).

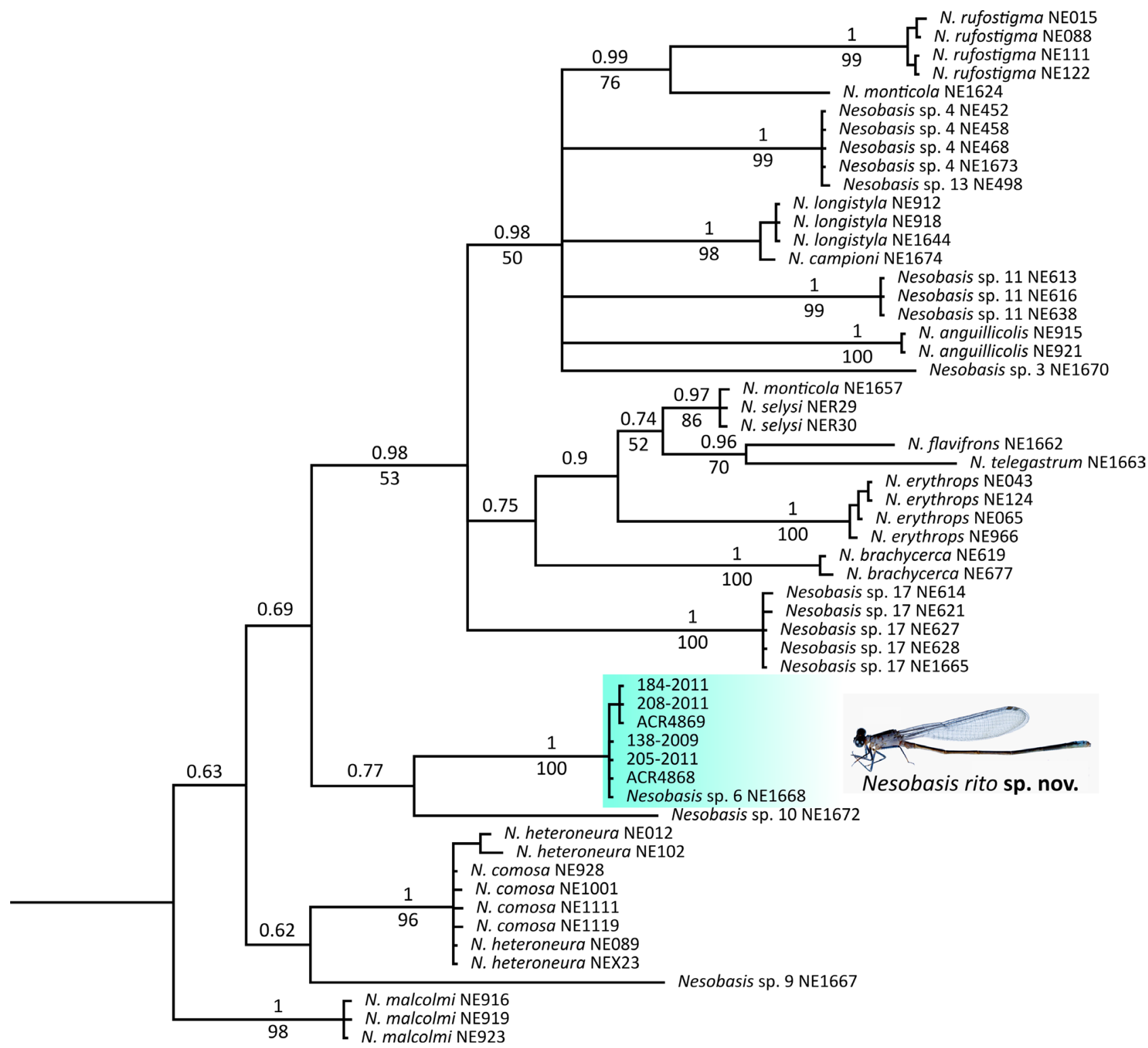

FIGURE 7. Phylogenetic tree representing the relationships among the Nesobasis species included in the present study for the COI mitochondrial marker. Values above/below branches represent BI posterior probability and ML bootstrap support values, respectively. Only bootstrap support values $\geq 50 \%$ are shown. Melanesobasis corniculata was used as the outgroup in these analyses (see main text and Table 1 for details) but, for the purposes of illustration, only the ingroup (i.e. Nesobasis) is shown here.

The species previously known as Nesobasis sp. nov. 6 and represented in our analyses by a single individual from Lorenzo-Carballa et al. (2019) (voucher ID NE1668; see Table 1), falls within the N. rito clade for both nuclear and mitochondrial DNA (Figs. 6-7). Also, the genetic distances between this individual and the specimens of $N$. rito sequenced in the present work are 0 and $0.1 \%$ for the nuclear and mitochondrial DNA, respectively, thus indicating that this individual is in fact a representative of $N$. rito. This would correlate to species $N$. sp. nov. 6 in Beatty et al. (2017), which is referred to as Uds2 in Beatty et al. (2007).

This species description is another step toward documenting the diversity of the genus Nesobasis in the Fiji Islands. A number of other congeneric species remain to be described, and the diversity of this group and the factors driving it need to be further explored. 


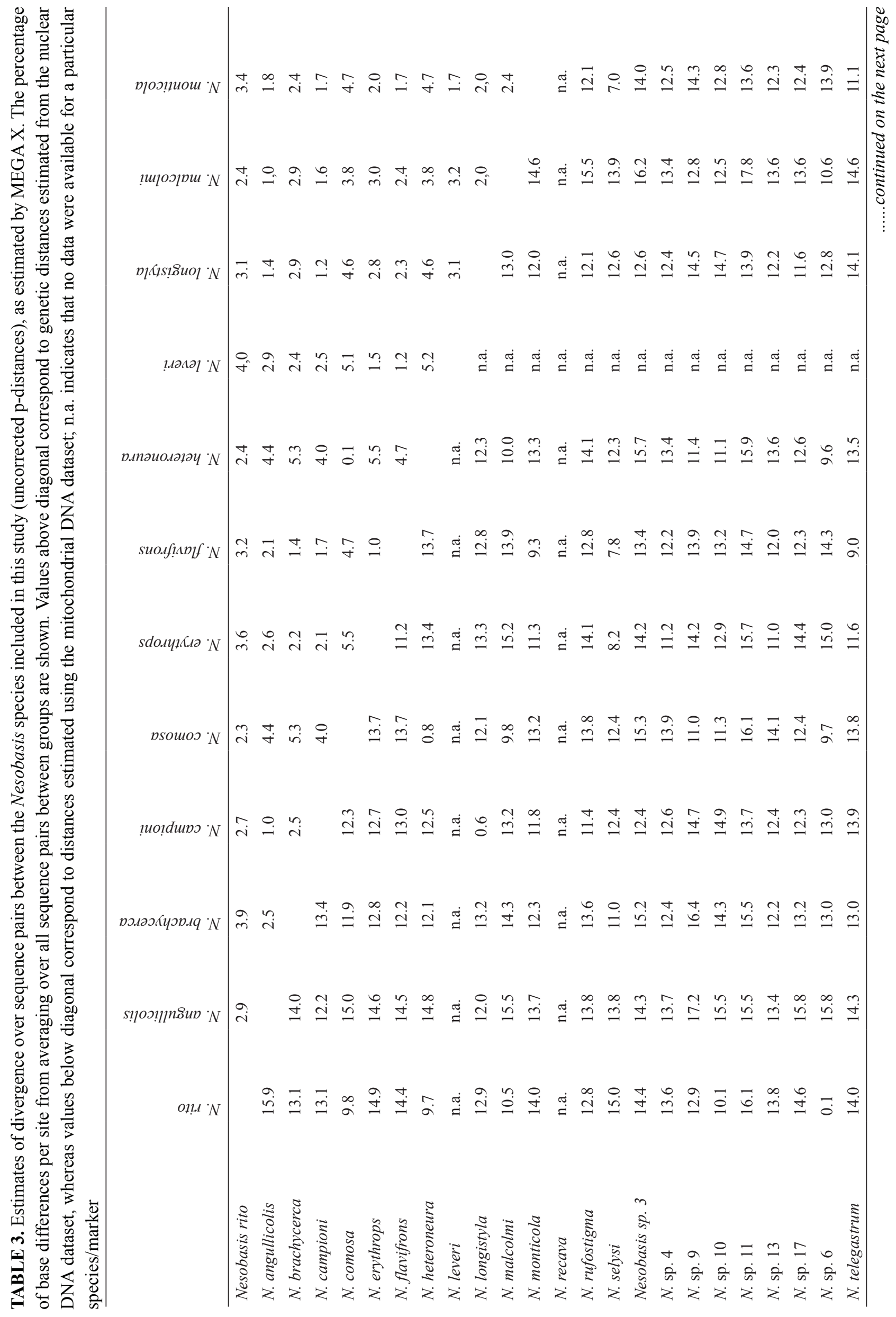




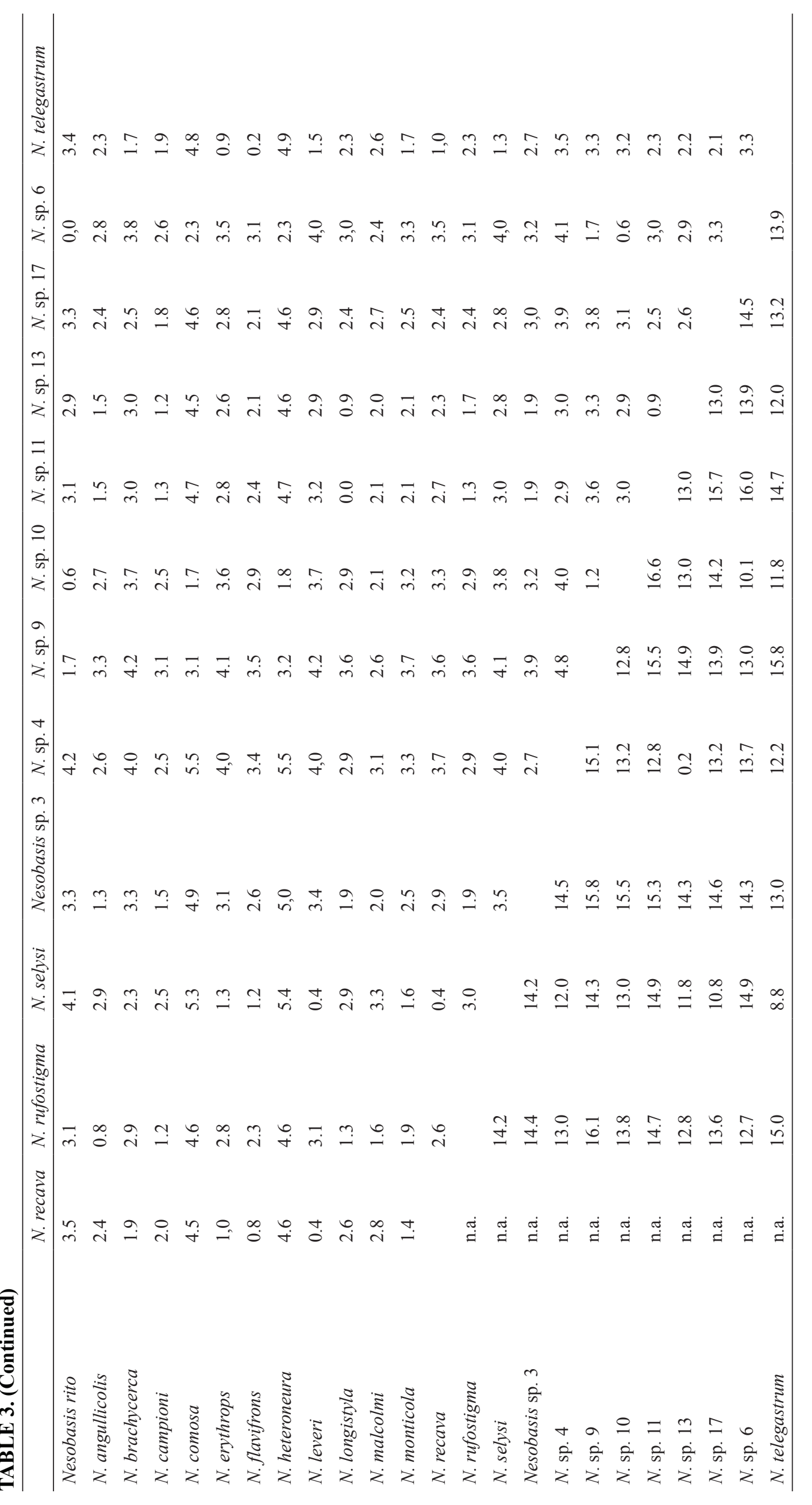




\section{Acknowledgments}

We warmly thank Tokasaya Cakacaka, Apaitia Liga and Alivereti Naikatini for their support in field and laboratory work and the Drawa villagers for their warm hospitality. This work was funded by grants CGL2014-53140-P and PGC2018-096656-B-I00 to ACR from MCIN/AEI/10.13039/501100011033 and from "ERDF A way of making Europe", by the "European Union".

\section{References}

Beatty, C.D., Van Gossum, H. \& Sherratt, T.N. (2007) Nesobasis species diversity and abundance: notes on an endemic genus of the island group of Fiji (Zygoptera: Coenagrionidae). Odonatologica, 36, $13-26$.

Beatty, C.D., Sánchez Herrera, M., Skevington, J.H., Rashed, A., Van Gossum, H., Kelso, S. \& Sherratt, T.N. (2017) Biogeography and systematics of endemic island damselflies: The Nesobasis and Melanesobasis (Odonata: Zygoptera) of Fiji. Ecology and Evolution, 7, 7117-7129.

https://doi.org/10.1002/ece3.3175

Bruzzese, D.J., Schuler, H., Wolfe, T.M., Glover, M.M., Mastroni, J., Doellman, M.M., Tait, C., Yee, W.L., Rull, J., Aluja, M., Hood, G.R., Goughnour, R., Stauffer, C., Nosil, P. \& Feder, J.L. (2021) Testing the potential contribution of Wolbachia to speciation when cytoplasmic incompatibility becomes associated with host-related reproductive isolation. Molecular Ecology, 1-16. [early view] https://doi.org/10.1111/mec. 16157

Donnelly, T.W. (1984) Melanesobasis gen. nov., a new genus of Fijian damselflies: a possible link between the platycnemidid Lieftinckia and certain coenagrionids (Zygoptera). Odonatologica, 13, 89-105.

Donnelly, T.W. (1990) The Fijian genus Nesobasis Part 1: Species of Viti Levu, Ovalau, and Kadavu (Odonata: Coenagrionidae). New Zealand Journal of Zoology, 17, 87-117. https://doi.org/10.1080/03014223.1990.10422587

Ferreira, S., Lorenzo-Carballa, M.O., Torres-Cambas, Y., Cordero-Rivera, A., Thompson, D.J. \& Watts, P.C. (2014) New EPIC nuclear DNA sequence markers to improve the resolution of phylogeographic studies of coenagrionids and other odonates. International Journal of Odonatology, 17, 135-147.

Futahashi, R. (2011) A revisional study of Japanese dragonflies based on DNA analysis (1). Tombo, 53, 67-74.

Garrison, R.W., von Ellenrieder, N. \& Louton, J.A. (2010) Damselfly genera of the New World. Johns Hopkins University Press, Baltimore, Maryland, $490 \mathrm{pp}$.

Huelsenbeck, J.P. \& Ronquist, F. (2001) MRBAYES: Bayesian inference of phylogenetic trees. Bioinformatics, 17, 754-755.

Katoh, K., Misawa, K. \& Miyata, T. (2002) MAFFT: a novel method for rapid multiple sequence alignment based on fast Fourier transform. Nucleic Acids Research, 30, 3059-3066.

Kimmins, D.E. (1943) A new species of Odonata from Fiji. Annals and Magazine of Natural History, Series 11, 10, 698-700. https://doi.org/10.1080/00222934308527384

Kumar, S., Strecher, G., Li, M., Knyaz, C. \& Tamura, K. (2018) MEGA X: Molecular Evolutionary Genetics Analysis across computing platforms. Molecular Biology and Evolution, 35, 1547-1549.

Lorenzo-Carballa, M.O. \& Cordero-Rivera, A. (2009) Thelytokous parthenogenesis in the damselfly Ischnura hastata (Odonata, Coenagrionidae): genetic mechanisms and lack of bacterial infection. Heredity, 103, 377-384.

Lorenzo-Carballa, M.O., Torres-Cambas, Y., Heaton, K., Hurst, G.D.D., Charlat, S., Sherratt, T.N., Van Gossum, H., CorderoRivera, A. \& Beatty, C.D. (2019) Widespread Wolbachia infection in an insular radiation of damselflies (Odonata, Coenagrionidae). Scientific Reports, 9, 11933. https://doi.org/10.1038/s41598-019-47954-3

Ronquist, F. \& Huelsenbeck, J.P. (2003) MrBayes 3: Bayesian phylogenetic inference under mixed models. Bioinformatics, 19, 1572-1574. https://doi.org/10.1093/bioinformatics/btg180

Selys-Longchamps, M.E. (1891) Causeries Entomologiques. No.3. Nesobasis Selys. (Nouveau sous-genre d'Agrionines.). Annales de la Société entomologique de Belgique, 35, 51-58.

Stamatakis, A. (2006) RAxML-VI-HPC: Maximum Likelihood-based Phylogenetic Analyses with Thousands of Taxa and Mixed Models. Bioinformatics, 22, 2688-2690.

Tillyard, R.J. (1924) The dragonflies (order Odonata) of Fiji, with special reference to a collection made by Mr. H. W. Simmonds, F. E. S., on the island of Viti Levu. Transactions of the Entomological Society of London, 1923, 305-346.

Van Gossum, H., Beatty, C.D., Charlat, S., Waqa, H., Markwell, T., Skevington, J.H., Tuiwawa, M. \& Sherratt, T.N. (2007) Male rarity and putative sex-role reversal in Fijian damselflies (Odonata). Journal of Tropical Ecology, 23, 591-598. https://doi.org/10.1017/S0266467407004373

Van Gossum, H., Beatty, C.D., Tokot'a, M. \& Sherratt, T.N. (2008) The Fijian Nesobasis: A further examination of species diversity and abundance (Zygoptera: Coenagrionidae). Odonatologica, 37, 235-245.

Werren, J.H., Baldo, L. \& Clark, M.E. (2008) Wolbachia: Master manipulators of invertebrate biology. Nature Reviews Microbiology, 6, 741-751.

https://doi.org/10.1038/nrmicro1969 